\title{
Continuous phase transition and critical behaviors of 3D black hole with torsion
}

\author{
Meng-Sen Ma, ${ }^{*}$ Fang Liu, and Ren Zhao ${ }^{\dagger}$ \\ Department of Physics, Shanxi Datong University, Datong 037009, China \\ Institute of Theoretical Physics, Shanxi Datong University, Datong 037009, China \\ we study the phase transition and the critical behavior of the BTZ black hole \\ with torsion obtained in $(1+2)$-dimensional Poincaré gauge theory. According to \\ Ehrenfest's classification, when the parameters in the theory are arranged properly \\ the BTZ black hole with torsion may posses the second order phase transition which \\ is also a smaller mass/larger mass black hole phase transition. Nevertheless, the \\ critical behavior is different from the one in the van der Waals liquid/gas system. \\ We also calculated the critical exponents of the relevant thermodynamic quantities, \\ which are the same as the ones obtained in the Hořava-Lifshitz black hole and the \\ Born-Infeld black hole. \\ PACS numbers: 04.70.-s, 05.70.Fh, 04.60.Kz
}

\section{INTRODUCTION}

The laws of black hole thermodynamics and the usual laws of thermodynamics have very similar forms. The good agreement indicates that black hole is also a thermodynamic system. Phase transitions and critical phenomena are important characteristics of usual thermodynamics. Thus, the natural question to ask is whether there also exists phase transition in the black hole thermodynamics. In fact, since the pioneering work of Davies[1] and the well-known Hawking-Page phase transition[2] were proposed, the question has been answered partly. The phase transitions and critical phenomena in four and higher dimensional AdS black holes have been studied extensively[3-6]. Recently, some interesting works on asymptotically anti-de Sitter black holes have been done, which show that there exists phase transition similar to the van der Waals liquid/gas phase transition[7-15].

The properties of black hole are relevant to the dimension of spacetime. Thus we want to know whether the similar phase transition exists for lower dimensional black holes. The key advantage of lower dimensional black holes lies in the simplicity of the construction. Although just mathematical abstraction, lower dimensional black holes can be applied to physical reality in some special cases. Hence it can be interesting to investigate the possibility that a lower dimensional black hole does exhibit a phase transition. BTZ black hole is an important solution of general relativity with negative cosmological constant in three-dimensional spacetime[16, 17]. The BTZ black hole is free of singularity and closely related with the recent developments in gravity, gauge theory and string theory[18].

In general relativity and many other theories of gravity, curvature plays an essential role , while torsion has received less attention. However, torsion also has its geometrical meaning and

*Electronic address: mengsenma@gmail.com

†Electronic address: zhao2969@sina.com 
plays some roles in gravitation theory. Since the 1970s, many theories of gravity with torsion have been proposed, such as Poincaré gauge gravity, de Sitter gauge gravity, teleparallel gravity, $f(T)$ gravity, etc. In particular, Mielke and Baekler proposed a model of three-dimensional gravity with torsion ( the MB model), which also has BTZ black hole as solution[19-23]. This model aroused the following research on the thermodynamics of the BTZ black hole with torsion (BTZT black hole for short) and AdS/CFT with torsion[24-26].

In [27] we have verified that for the BTZT black hole phase transition may exist. In this paper we will investigate the type of the phase transition and calculate the critical exponents. Although the BTZ solution is the same as the one obtained in GR, the different actions will make their thermodynamics very different. The modified action in the MB model will modify the conserved charges such as mass and angular momentum. Correspondingly the entropy of BTZ black hole and the first law of black hole thermodynamics will also been changed. It is shown that the heat capacity of the BTZ black hole in the MB model is not always positive any more, but changes signs at some points and may diverge at the critical point. Thus for the BTZT black hole in the MB model phase transition exists. According to Ehrenfest's classification we also consider the Gibbs free energy, the isothermal compressibility and the expansion coefficient as functions of temperature. It is shown that the kind of phase transition for the BTZT black hole belong to the second-order one or continuous one.

The paper is arranged as follows: in the next section we simply introduce the MB model and its BTZ-like solution and the corresponding thermodynamic quantities. In section 3 according to Ehrenfest's classification we will analyze the type of the phase transition of the BTZT black hole in the extended phase space. In section 4 the case with non-extended phase space will be discussed and the critical behaviors are investigated. We make some concluding remarks in section 5 .

\section{MIELKE-BAEKLER MODEL AND THE 3D BLACK HOLE WITH TORSION}

First we should review the topological three-dimensional gravity model with torsion proposed by Mielke and Baekler[19, 20], which is a natural generalization of Riemannian GR with a cosmological constant. Defining curvature and torsion 2 -forms out of $\omega^{a}{ }_{b}$ and coframe $e^{a}$ by

$$
\begin{aligned}
T^{a} & =d e^{a}+\omega^{a}{ }_{b} \wedge e^{b}, \\
R_{b}^{a} & =d \omega_{b}^{a}+\omega^{a}{ }_{c} \wedge \omega_{b}^{c},
\end{aligned}
$$

the gravitational action is written as

$$
I=\int 2 \chi e^{a} \wedge R_{a}-\frac{\Lambda}{3} \epsilon_{a b c} e^{a} \wedge e^{b} \wedge e^{c}+\alpha_{3}\left(\omega^{a} \wedge d \omega_{a}+\frac{1}{3} \varepsilon_{a b c} \omega^{a} \wedge \omega^{b} \wedge \omega^{c}\right)+\alpha_{4} e^{a} \wedge T_{a}
$$

where the dual expression, $R_{a}$ and $\omega_{a}$ are defined by $R^{a b}=\epsilon^{a b c} R_{c}$ and $\omega^{a b}=\epsilon^{a b c} \omega_{c}$. In Eq.(2.3) the first term corresponds to the Einstein-Cartan action, with $\chi=\frac{1}{16 \pi G}$. The second one is the cosmological term. The last two terms are the Chern-Simons term and the Nieh-Yan term, which should be given particular attention.

The Nieh-Yan(N-Y) form is a special 2-form only for the Riemann-Cartan geometry[28, 29]. On the four-dimensional manifold $M$ it can be written as

$$
\begin{aligned}
& N=T^{a} \wedge T_{a}+R_{a b} \wedge e^{a} \wedge e^{b}=d Q_{N Y} \\
& Q_{N Y}=e^{a} \wedge T_{a}
\end{aligned}
$$


The N-Y form is a kind of Chern-Simons form and will have its application to manifolds with boundaries and reflect the role of torsion in geometry.

After variation to $\omega^{a}{ }_{b}$ and $e^{a}$, two vacuum equations can be obtained from the MB action $(2.3)$

$$
\begin{aligned}
T^{a} & =\frac{p}{2} \varepsilon_{b c}^{a} e^{b} \wedge e^{c}, \\
R^{a} & =\frac{q}{2} \varepsilon_{b c}^{a} e^{b} \wedge e^{c},
\end{aligned}
$$

with the two constant coefficients $p, q$ defined by $p=\frac{\alpha_{3} \Lambda+\alpha_{4} \chi}{\alpha_{3} \alpha_{4}-\chi^{2}}$ and $q=-\frac{\alpha_{4}^{2}+\chi \Lambda}{\alpha_{3} \alpha_{4}-\chi^{2}}$.

The curvatures in Einstein-Cartan geometry can be connected to their counterparts in Riemannian geometry. In particular, in three-dimensional spacetime, the equations above can be simplified to equations without torsion

$$
\tilde{R}^{a}=\frac{\Lambda_{e f f}}{2} \varepsilon_{b c}^{a} e^{b} \wedge e^{c}
$$

where $\tilde{R}^{a}$ is the curvature without torsion and $\Lambda_{\text {eff }}=q-\frac{1}{4} p^{2}$ is the effective cosmological constant. One can let $\Lambda_{e f f}=-\frac{1}{l^{2}}<0$ to construct an asymptotically anti-de Sitter space.

As in the three-dimensional Einstein equation, Eq.(2.7) has the well-known BTZ solution. But in this case, torsion is contained in the gravitational action. The metric is

$$
d s^{2}=-N(r)^{2} d t^{2}+\frac{1}{N(r)^{2}} d r^{2}+r^{2}\left(d \phi+N_{\phi} d t\right)^{2}
$$

where

$$
N(r)^{2}=\frac{r^{2}}{l^{2}}-M_{0}+\frac{J_{0}^{2}}{4 r^{2}}, \quad N_{\phi}(r)=\frac{J_{0}}{2 r^{2}} .
$$

Here we have considered $8 G=1$. This metric is the same as the one in GR, except that $l=1 / \sqrt{-\Lambda_{\text {eff }}}$ here and a constant torsion[21]. For this metric there are two horizons: the outer one $r_{+}$and the inner one $r_{-}$. From $N^{2}(r)=0$, one can obtain the expressions of both horizons:

$$
r_{ \pm}^{2}=\frac{M_{0} l^{2}}{2}(1 \pm \Delta), \quad \Delta=\left[1-\left(J_{0} / M_{0} l\right)^{2}\right]^{1 / 2}
$$

Conversely, $M_{0}$ and $J_{0}$ can be expressed as follows:

$$
M_{0}=\frac{r_{+}^{2}+r_{-}^{2}}{l^{2}}, \quad J_{0}=\frac{2 r_{+} r_{-}}{l} .
$$

Hawking radiation is just a kinematic effect, which only depends on the event horizon and is irrelevant to the dynamical equations and the gravitational theories. Therefore the temperature of BTZ black hole in the MB model has the similar form as in GR, which is

$$
T=\frac{r_{+}^{2}-r_{-}^{2}}{2 \pi l^{2} r_{+}}
$$

Certainly because of the existence of $l$, the temperature is relevant to the coefficients $\alpha_{3}, \alpha_{4}, \Lambda$ of MB Lagrangian. Define

$$
\Omega_{H}=-\left.\frac{g_{t \phi}}{g_{\phi \phi}}\right|_{r_{+}}=\frac{J_{0}}{2 r_{+}^{2}}
$$

which can be regarded as the angular velocity of BTZ black hole. 
Because of the existence of the topological terms, the asymptotically behavior is different from the one for Einstein-Cartan theory. Blagojevic et.al have proved that the gravitational conserved charges in the MB model should be[24, 25, 30]

$$
M=M_{0}+2 \pi \alpha_{3}\left(\frac{p M_{0}}{2}-\frac{J_{0}}{l^{2}}\right)=a M_{0}-\frac{b}{l^{2}} J_{0}, \quad J=J_{0}+2 \pi \alpha_{3}\left(\frac{p J_{0}}{2}-M_{0}\right)=a J_{0}-b M_{0}
$$

where we have defined $a=1+\pi \alpha_{3} p, b=2 \pi \alpha_{3}$. Obviously when $\alpha_{3}=0$ they will return to their conventional interpretation as energy and angular momentum, as with the BTZ metric in general relativity.

Correspondingly the entropy can be derived

$$
S=4 \pi r_{+}+4 \pi^{2} \alpha_{3}\left(p r_{+}-\frac{2 r_{-}}{l}\right)=4 \pi\left(a r_{+}-\frac{b}{l} r_{-}\right)
$$

It differs from the Bekenstein-Hawking result by an additional term and will coincides with Solodukhin's result if $p=0[31]$. Black hole entropy is not always equated with one quarter of the event horizon area. In fact it is related to the gravitational theory under consideration. It can be easily verified that in the MB model the entropy, temperature, and the conserved charges not only satisfy the first law of thermodynamics

$$
d M=T d S+\Omega_{H} d J
$$

but also fulfill the Smarr-like formula

$$
M=\frac{1}{2} T S+\Omega_{H} J
$$

This further implicates that with torsion the BTZ black hole can still be treated as a thermodynamic system and the thermodynamic laws still hold. It should be noted that in the expression of the entropy of the BTZ black hole no torsion exists explicitly, only $\alpha_{4}$ in $p$ implicitly. In particular, when $\alpha_{3}=0$ the entropy in Eq.(2.15) returns to the usual BTZ black hole entropy. It means that the N-Y term $\alpha_{4} e^{a} \wedge T_{a}$ influences the conserved charges and the exact form of entropy only when the CS term exists.

\section{PHASE TRANSITION IN EXTENDED PHASE SPACE}

Ehrenfest had ever attempted to classify the phase transitions . Phase transitions connected with an entropy discontinuity are called discontinuous or first order phase transitions, and phase transitions where the entropy is continuous are called continuous or second/higher order phase transitions. More precisely, for the first-order phase transition the Gibbs free energy $G(T, P, \ldots)$ should be continuous and its first derivative with respect to the external fields:

$$
S=-\left.\frac{\partial G}{\partial T}\right|_{(P, \ldots)}, \quad V=\left.\frac{\partial G}{\partial P}\right|_{(T, \ldots)}
$$

are discontinuous at the phase transition points.

For the second-order phase transition the Gibbs free energy $G(T, P, \ldots)$ and its first derivative are both continuous, but the second derivative of $G$ will diverge at the phase transition points like the specific heat $C_{P}$, the compressibility $\kappa$, the expansion coefficient $\alpha$ :

$$
C_{P}=\left.T \frac{\partial S}{\partial T}\right|_{P}=-\left.T \frac{\partial^{2} G}{\partial T^{2}}\right|_{P}, \kappa=-\left.\frac{1}{V} \frac{\partial V}{\partial P}\right|_{T}=-\left.\frac{1}{V} \frac{\partial^{2} G}{\partial P^{2}}\right|_{T}, \alpha=-\left.\frac{1}{V} \frac{\partial V}{\partial T}\right|_{P}=-\frac{1}{V} \frac{\partial^{2} G}{\partial P \partial T}
$$


In this sense, because the heat capacity is always positive, there is no second order phase transition for BTZ black hole obtained in GR. This property of BTZ black hole can also be verified by the method of thermodynamic curvature[32, 33].

To utilize Ehrenfest's classification, we consider variable cosmological constant and relate it to the pressure[10, 11, 14, 15, 34, 35]. The first law of thermodynamics for the BTZT black hole should be

$$
d M=T d S+\Omega d J+V d P
$$

where $P=\frac{1}{8 \pi l^{2}}=-\frac{\Lambda_{e f f}}{8 \pi}$, and $V=\left.\frac{\partial M}{\partial P}\right|_{S, J}$ is the corresponding thermodynamic volume. Therefore the mass of black hole is no more internal energy, but should be interpreted as the thermodynamic enthalpy, namely $H=M(S, P, J)[10,11,34,35]$. The first law of black hole thermodynamics represented by the internal energy $U(S, V, J)$ reads

$$
d U=T d S+\Omega d J-P d V
$$

where $U=H-P V$.

For BTZT black hole one can express mass $M$ as functions of $S, J, P$, which are

$$
H_{ \pm}=M_{ \pm}=\frac{1}{8 \pi^{2} b^{2}}\left[a S^{2}+8 \pi^{2} a b J \pm S \sqrt{\left(a^{2}-8 \pi b^{2} P\right)\left(S^{2}+16 \pi^{2} b J\right)}\right]
$$

One can substitute the expressions of $S, M, J$ into Eq.(3.5) to test and verify it directly. This result can also be verified easily by differentiating the mass $M_{ \pm}$with the entropy $S$ to get the Hawking temperature, Eq.(2.12).

It should be noted that when $M$ is expressed with $r_{+}, r_{-}, l$, its form is unique. When we express it with the thermodynamic quantities, two different forms $M_{ \pm}$appear. In fact $M_{ \pm}$ depend on the relation between $a l$ and $b$. They are established under different conditions:

- $b^{2} \leq a^{2} l^{2}$ or $b^{2} \leq \frac{a^{2}}{8 \pi P}$, the expression $M_{-}$is right. At this time, to keep the expression in square root have physical meaning, $S^{2}+16 \pi^{2} b J \geq 0$ should also be satisfied.

- $b^{2} \geq a^{2} l^{2}$ or $b^{2} \geq \frac{a^{2}}{8 \pi P}$, the expression $M_{+}$should be used. Similarly, to keep the expression in square root have physical meaning, $S^{2}+16 \pi^{2} b J \leq 0$ should be satisfied.

According to Eqs.(2.14),(2.15), when $|b| \geq|a| l, M, J, S$ may be negative. In fact for gravities with higher derivative terms there is the possibility for negative entropy and energy which depend on the parameters of higher derivative terms[36]. Although black holes behave as thermodynamic systems, they also show some exotic behaviors, the most known one is the entropy of black holes is proportional to area and not the volume. Therefore it is understandable if black holes exhibit some strange thermodynamic properties. Below we will show that the condition $|b| \geq|a| l$ is the key for the BTZT black hole to have phase transition.

The temperature of BTZT black hole can be evaluated according to $S, P, J$ :

$$
T_{-}(S, J, P)=\left.\frac{\partial M_{-}}{\partial S}\right|_{P, J} \text { or } \quad T_{+}(S, J, P)=\left.\frac{\partial M_{+}}{\partial S}\right|_{P, J}
$$

They look different when expressed with the thermodynamic quantities $S, P, J$ because they correspond to different conditions for the parameters $a, b, l$. When replacing the thermodynamic variables $(S, J, P)$ with the geometric ones $\left(r_{+}, r_{-}, l\right)$, the two expressions can be unified and Eq.(2.12) can turn up. 
According to Eqs.(3.5),(3.6), the specific heats at constant pressure and constant angular momentum can be calculated easily:

$$
C_{-}=\left.\frac{\partial M_{-}}{\partial T-}\right|_{P, J}=T_{-} \frac{1}{\left.\frac{\partial T_{-}}{\partial S}\right|_{P, J}}, \quad C_{+}=\left.\frac{\partial M_{+}}{\partial T+}\right|_{P, J}=T_{+} \frac{1}{\left.\frac{\partial T_{+}}{\partial S}\right|_{P, J}}
$$

With the geometric quantities, the heat capacity can be written as

$$
C_{P}=C_{ \pm}=\frac{4 \pi r_{+}^{2}\left(r_{+}^{2}-r_{-}^{2}\right)\left(b^{2}-a^{2} l^{2}\right)}{l\left(b r_{-}\left(3 r_{+}^{2}+r_{-}^{2}\right)-a l r_{+}\left(r_{+}^{2}+3 r_{-}^{2}\right)\right)}
$$

It is more appropriate to study the phase transition of the BTZT black holes according to geometric quantities $r_{+}, r_{-}$. Because the conditions $r_{+} \geq r_{-}, M_{0}>0, J_{0}>0$ must be fulfilled. If employing the thermodynamic quantities completely, one may omits these conditions. Below we will set $l=1$ and analyze the phase transition of the BTZT black hole numerically. According to Ehrenfest's classification, we should first derive the Gibbs free energy $G$ :

$$
G=H-T S=M-T S
$$

\section{A. $\quad a^{2} l^{2} \geq b^{2}$}

One can easily plot the $G_{-}-T, S-T, C_{-}-T$ curves as shown in Fig.1. Obviously $G_{-}, S, C_{-}$are all continuous function of temperature $T$. No turning point and divergence turn up, which means no second-order phase transition happens in this case. This conclusion can also be supported by the method of thermodynamic curvature[33, 37].
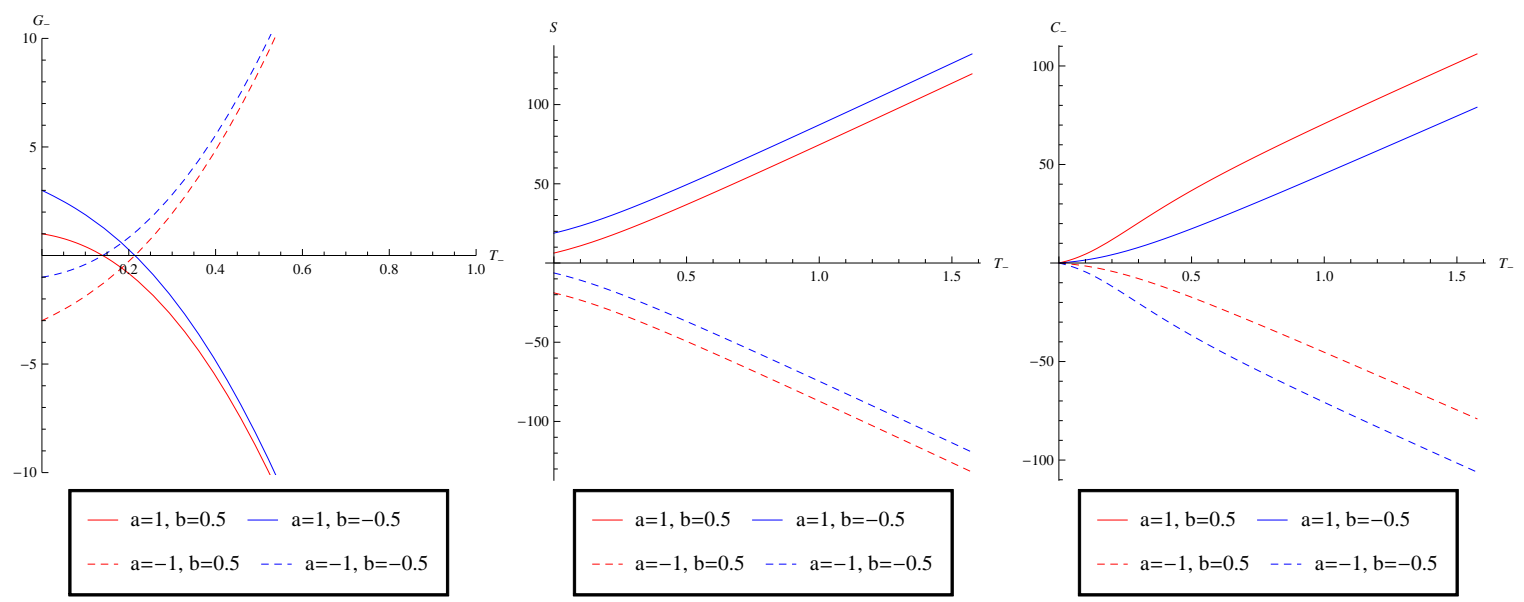

FIG. 1: The Gibbs free energy, entropy and heat capacity at constant pressure as functions of temperature for BTZT black hole for the choices of $l=1, r_{-}=1, a= \pm 1, b= \pm 0.5$ and $r_{+} \geq r_{-}$. For $C_{-}$ no divergent point exists.

\section{B. $\quad a^{2} l^{2} \leq b^{2}$}

In this case we first plot the $C_{+}-r_{+}$curves for different values of $a, b$. In Fig.2(a) and Fig.2(b) we show that there is no divergent point when $a, b$ take opposite signs. The phase transition 
may happen only when $a, b$ are both positive or negative. Under the given conditions one can easily derive the position of the divergent point, $r_{c} \approx 5.522$. Obviously the phase transition is a smaller mass/larger mass black hole phase transition. When $a>0, b>0$, the smaller black hole is stable because the heat capacity is positive, when $a<0, b<0$, the other way around, the larger black hole is stable.

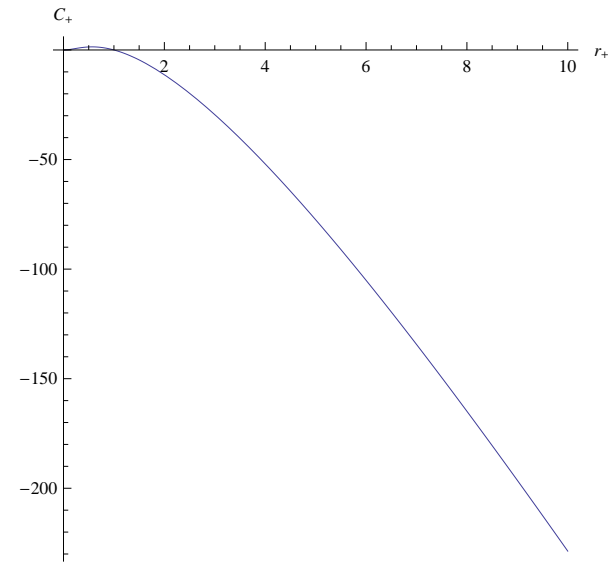

(a) $a=1, b=-2$

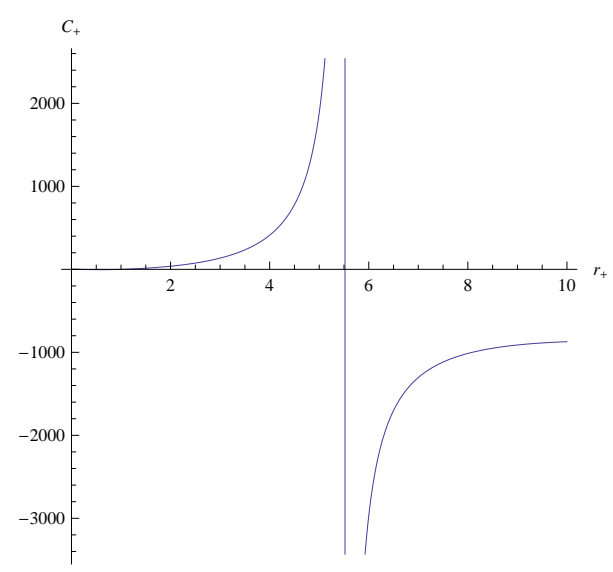

(c) $a=1, b=2$

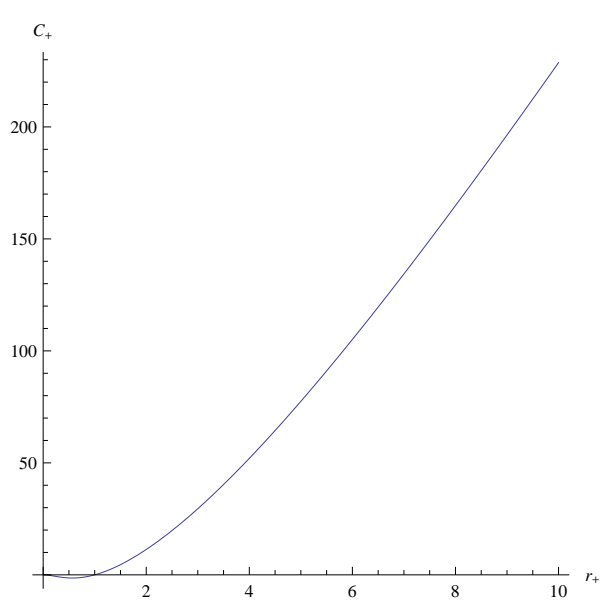

(b) $a=-1, b=2$

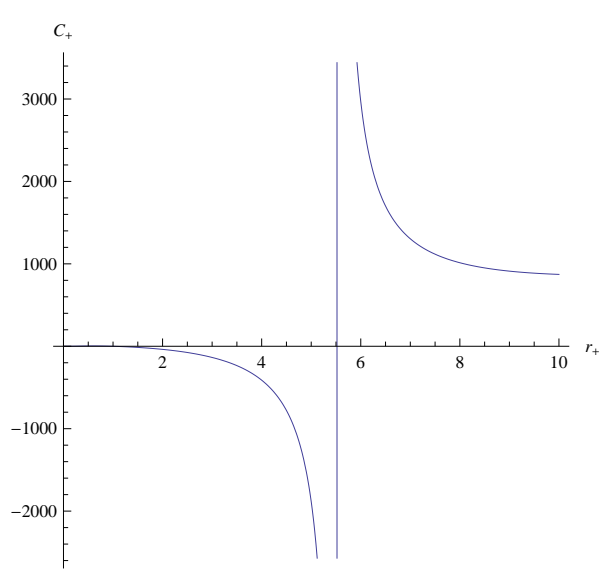

(d) $a=-1, b=-2$

FIG. 2: The heat capacity at constant pressure and angular momentum as functions of $r_{+}$for BTZT black hole for the choices of $l=1, r_{-}=1, a= \pm 1, b= \pm 2$. In the cases $(c),(d)$ there exist the divergent points for $C_{+}$.

One should further analyze the value of $M, J, S$. We only consider the two cases which have the phase transitions. In Fig.3 it is shown that under the condition $a^{2} l^{2} \leq b^{2}$ when $a, b$ are both positive, the corrected mass $M$ and the entropy $S$ are positive at the divergent point $r_{c}$, while $J$ is negative; for the case with negative $a, b$, the situation is just the opposite. Thus we conclude that for the BTZT black hole if the phase transition can happen, the $M, J, S$ cannot be all positive.

Now we investigate the type of the phase transition for the BTZT black hole according to Ehrenfest's classification. To calculate the isothermal compressibility $\kappa$ and the expansion coefficient $\alpha$ we should first obtain the thermodynamic volume.

$$
V_{+}=\left.\frac{\partial M_{+}}{\partial P}\right|_{S, J}, \quad V_{-}=\left.\frac{\partial M_{-}}{\partial P}\right|_{S, J}
$$




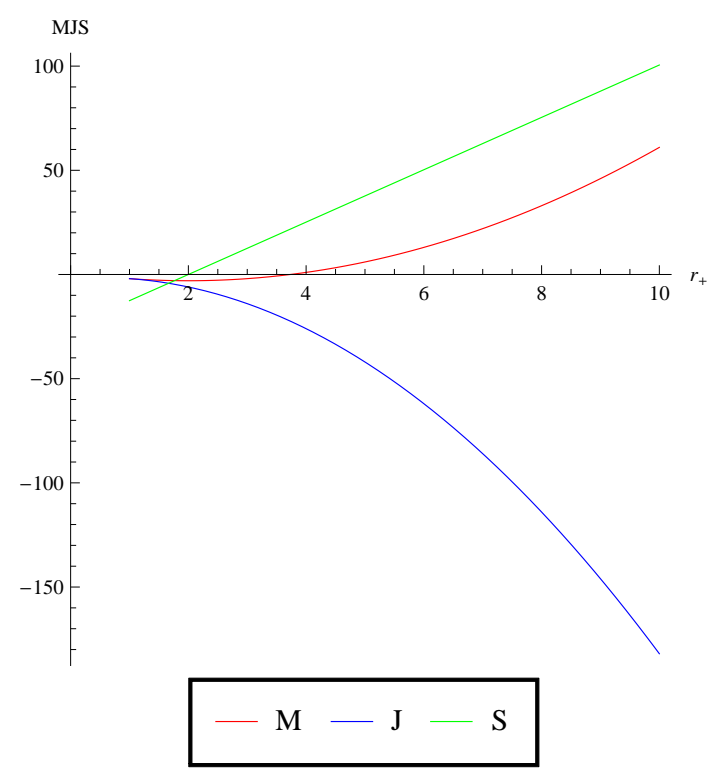

(a) $a=1, b=2$

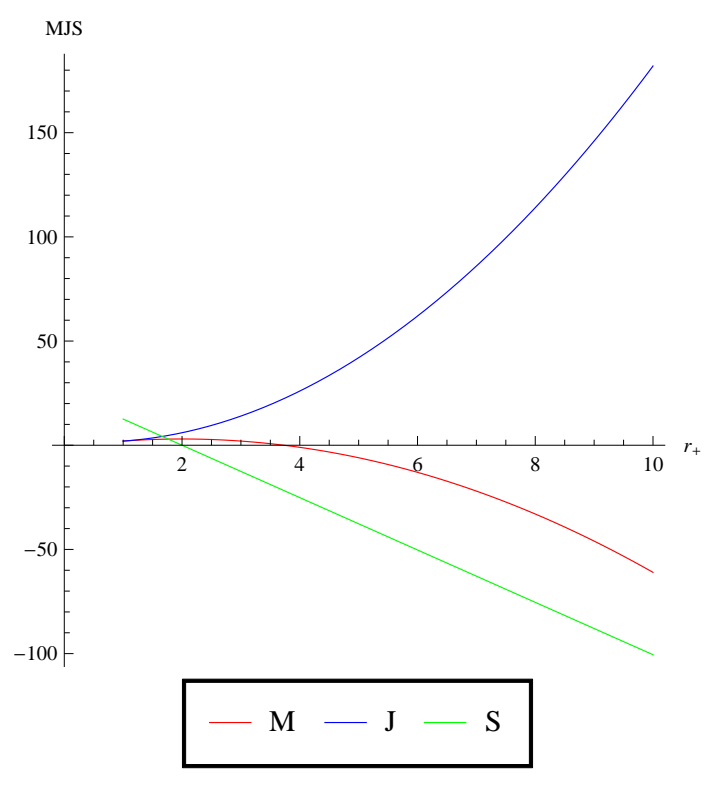

(b) $a=-1, b=-2$

FIG. 3: The conserved charges $M, J$, and the entropy as functions of $r_{+}$for BTZT black hole for the choices of $l=1, r_{-}=1, a= \pm 1, b= \pm 2$ and $r_{+} \geq r_{-}$.

Although $M_{+}$and $M_{-}$are different, when considering the conditions for $M_{ \pm}$one can find that

$$
V=V_{+}=V_{-}=\frac{S}{2 \pi} \sqrt{\frac{16 \pi^{2} b J+S^{2}}{a^{2}-8 \pi b^{2} P}}
$$

Inversely, one can derive the pressure

$$
P=\frac{-16 \pi^{2} b J S^{2}-S^{4}+4 \pi^{2} a^{2} V^{2}}{32 \pi^{3} b^{2} V^{2}}
$$

which must be greater than zero. Thus $4 \pi^{2} a^{2} V^{2}-16 \pi^{2} b J S^{2}-S^{4} \geq 0$ should be satisfied. Because $V=V(S, P, J)$,

$$
\left.\frac{\partial V}{\partial P}\right|_{T}=\left.\frac{\partial V}{\partial P}\right|_{S}+\left.\frac{\partial V}{\partial S} \frac{\partial S}{\partial P}\right|_{T}
$$

and

$$
\left.\frac{\partial V}{\partial T}\right|_{P}=\left.\frac{\partial V}{\partial S} \frac{\partial S}{\partial T}\right|_{P}
$$

According to the above equations one can derive the isothermal compressibility $\kappa$ and the expansion coefficient $\alpha$. We can plot the curves of $G-T, S-T, C-T, \kappa-T, \alpha-T$ in Fig.4. Similarly, only when the parameters $a, b$ have the same sign, the phase transition for the BTZT black hole can turn up. The critical temperature lies at $T_{c}=0.85$. As is shown in the figure, the Gibbs free energy and the entropy are continuous functions of temperature. While the heat capacity, the isothermal compressibility and the expansion coefficient all diverge at the critical point. Therefore the phase transition at this critical point is the second-order phase transition or continuous one.

According to Eqs.(3.11), (3.12) and $U=H-P V$, we can obtain the internal energy

$$
U=U(S, V, J)=-\frac{\left(S^{2}-2 \pi a V\right)\left(-2 \pi a V+16 \pi^{2} b J+S^{2}\right)}{32 \pi^{3} b^{2} V}
$$




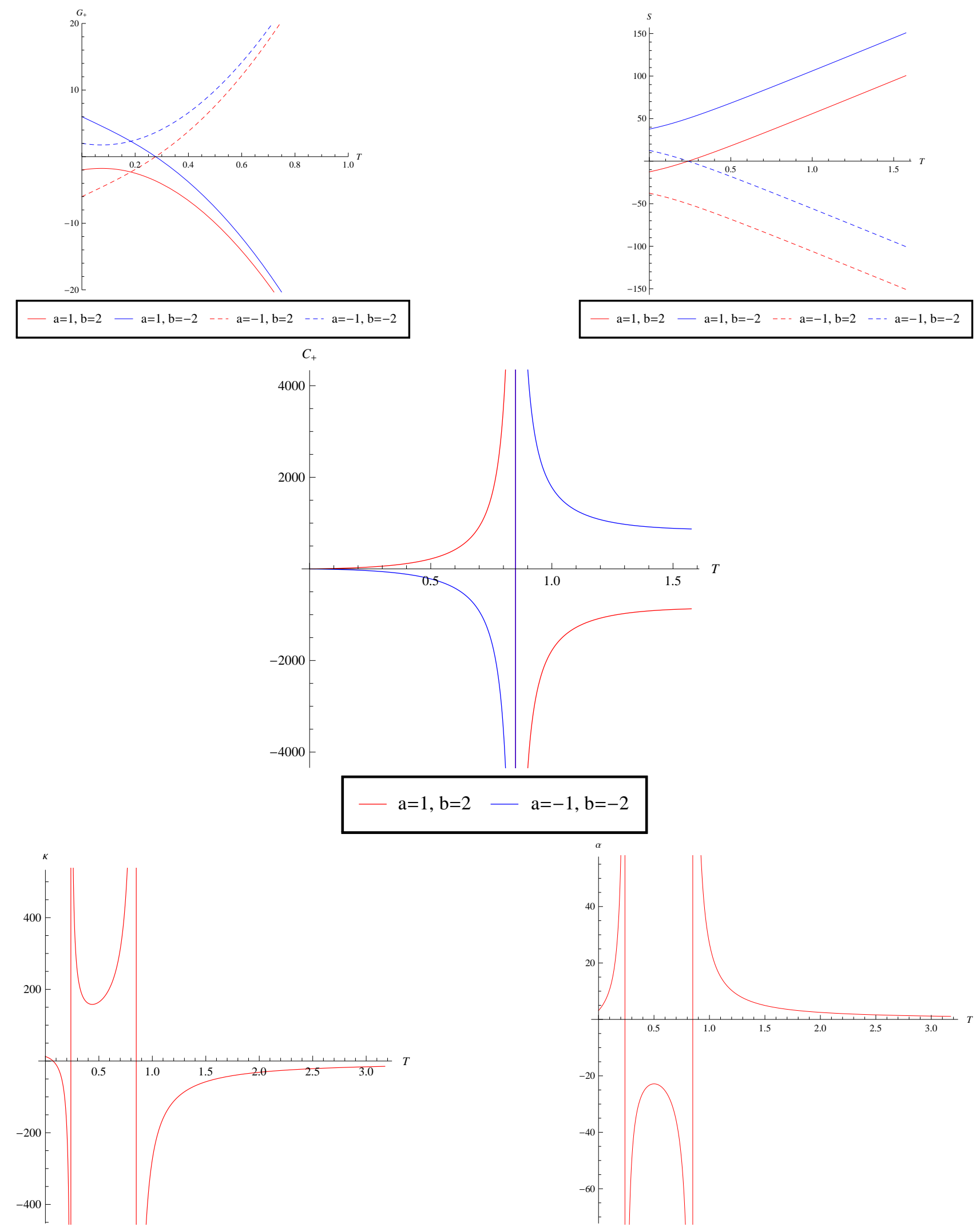

FIG. 4: The Gibbs free energy, entropy, the heat capacity at constant pressure and the isothermal compressibility and the expansion coefficient as functions of temperature for BTZT black hole for the choices of $l=1, r_{-}=1, a= \pm 1, b= \pm 2$ and $r_{+} \geq r_{-}$. For the two cases $a=1, b=2$ and $a=-1, b=-2$, the $\kappa-T$ and the $\alpha-T$ curves are both concurrent respectively. 
From which one can easily derive the temperature as functions of $S, V, J$ :

$$
T(S, V, J)=\frac{S\left(2 a \pi V-8 \pi^{2} b J-S^{2}\right)}{8 b^{2} \pi^{3} V}
$$

From Eq.(3.12) and Eq.(3.16), one can derive the equation of state between the pressure $P$, the temperature $T$ and the volume $V$ by eliminating $S$. Thus one can obtain the pressure $P$ as function of $V, T, J$ in principle. But the expression is too lengthy and obviously it will depend on the value of $J$. Below we will analyze the $P-V$ relation by means of the static scaling law[38]. Dimensional analysis implies that the $P$ and $T$ are both homogeneous functions of the variables $S, V, J$, since $P \rightarrow P, T \rightarrow \lambda T$ when $V \rightarrow \lambda^{2} V, S \rightarrow \lambda S, J \rightarrow \lambda^{2} J$. Thus $P$ and $T$ are in fact the functions of two independent variables. The same logic also applies to the internal energy $U$. So we can take advantage of the scaling character to redefine the functions and the variables. One can take

$$
t=\frac{T}{S}, \quad p=P, \quad v=\frac{V}{S^{2}}, \quad j=\frac{J}{S^{2}}
$$

In this way the entropy $S$ can be eliminated in Eqs.(3.12), (3.16) and they are simplified to be

$$
\begin{aligned}
& t=\frac{2 \pi v-8 \pi^{2} \beta j-1}{8 \pi^{3} \beta^{2} v} \\
& p=-\frac{16 \pi^{2} \beta j+1-4 \pi^{2} v^{2}}{32 \pi^{3} \beta^{2} v^{2}}
\end{aligned}
$$

Further removing the $j$ and combing the two equations together, one can get

$$
p=\frac{t}{2 v}+\frac{1}{8 b^{2} \pi^{2}}-\frac{1}{8 b^{2} \pi^{2} v}+\frac{1}{32 b^{2} \pi^{3} v^{2}}
$$

The great advantage of the above relation lies at it is irrelevant to $J$ and is more universal. Although the $p-v$ structure is not the same as the $P-V$ structure, it can reflect some properties of the system. The critical point occurs at the point where

$$
\frac{\partial p}{\partial v}=0, \quad \frac{\partial^{2} p}{\partial v^{2}}=0
$$

But unfortunately, the above equations do not have solutions. One can also easily plot the $p-v$ curves of Eq.(3.19) at different temperatures. We can draw conclusions that for the BTZT black hole there is no the similar phase structure and critical behavior to the van der Waals liquid-gas system.

\section{CRITICAL BEHAVIORS IN NON-EXTENDED PHASE SPACE}

In the non-extended phase space, the $l$ or $\Lambda$ should be considered as constant. Thus Eq.(3.5) is modified to

$$
M_{ \pm}=\frac{1}{8 \pi^{2} \beta^{2}}\left[a S^{2}+8 \pi^{2} a b J \pm \frac{S}{l} \sqrt{\left(a^{2} l^{2}-b^{2}\right)\left(S^{2}+16 \pi^{2} b J\right)}\right]
$$

When considering the $J-\Omega$ relations some rotating black holes such as Kerr-AdS black hole will exhibit similar critical behavior to the van der Waals liquid-gas system[39, 40]. Thus we analyze for the BTZT black hole whether there are similar conclusion. The $J-\Omega$ relation can be easily obtained: 


$$
J=-\frac{4 \pi^{2} T^{2}\left(-2 a l^{2} \Omega+b l^{2} \Omega^{2}+b\right)}{\left(l^{2} \Omega^{2}-1\right)^{2}}
$$

One may try to derive the critical point according to

$$
\left.\frac{\partial J}{\partial \Omega}\right|_{T}=0,\left.\quad \frac{\partial^{2} J}{\partial \Omega^{2}}\right|_{T}=0
$$

But it can be easily verified that also no solution exists. In this case the heat capacity $C$ is the same as Eq.(3.8). However, the isothermal compressibility should be defined as[40]

$$
\kappa_{T}=\left.\frac{\partial \Omega}{\partial J}\right|_{T}=-\frac{\left(l^{2} \Omega^{2}-1\right)^{3}}{8 \pi^{2} l^{2} T^{2}\left(3 a l^{2} \Omega^{2}+a-b l^{2} \Omega^{3}-3 b \Omega\right)}
$$

We plot the $\kappa_{T}-T$ curves as in Fig.5. In this case the critical temperature still lies at $T_{c}=0.85$.

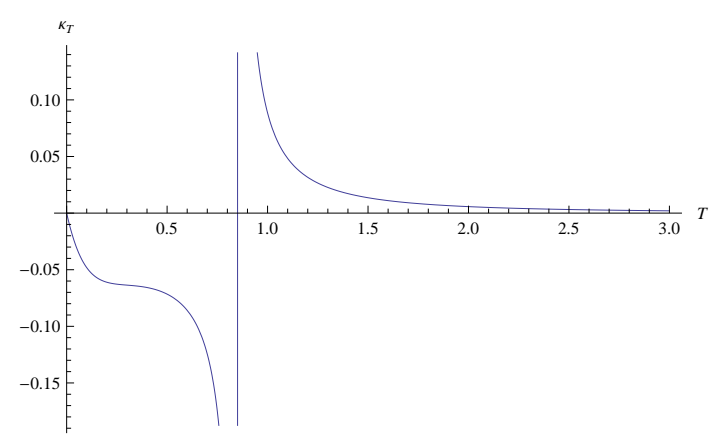

(a) $a=1, b=2$

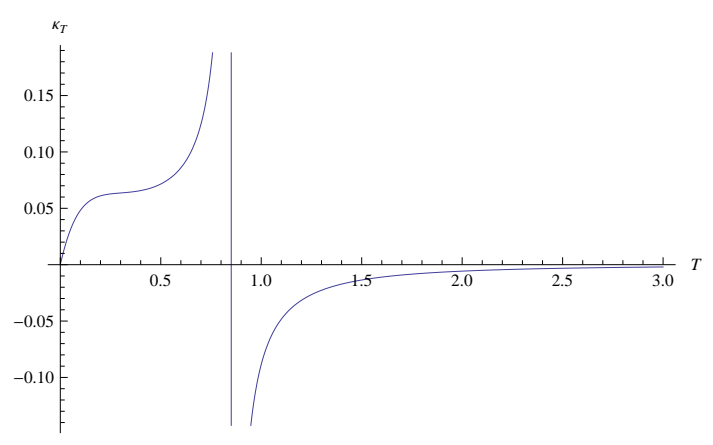

(b) $a=-1, b=-2$

FIG. 5: The isothermal compressibility as functions of temperature for BTZT black hole for the choices of $l=1, r_{-}=1, a= \pm 1, b= \pm 2$.

In order to see the thermodynamic behavior near the critical point, the critical exponents can be introduced as

$$
\begin{aligned}
& J-J_{c} \sim\left|\Omega-\Omega_{c}\right|^{\delta}, \quad \Omega-\Omega_{c} \sim\left|T-T_{c}\right|^{\beta} \\
& C_{J} \sim\left|T-T_{c}\right|^{-\alpha}, \quad \kappa_{T} \sim\left|T-T_{c}\right|^{-\gamma}
\end{aligned}
$$

From Eq.(4.2), at the critical point $T=T_{c}$, the first derivative of $J$ over $\Omega$ satisfy

$$
\left.\frac{\partial J}{\partial \Omega}\right|_{T_{c}}=0
$$

But the second derivative can be calculated as

$$
\left.\frac{\partial^{2} J}{\partial \Omega^{2}}\right|_{T_{c}}= \pm 322.187 \neq 0
$$

for the two cases $a=1, b=2, l=1$ and $a=-1, b=-2, l=1$. Thus

$$
J-J_{c}=\left.\frac{\partial^{2} J}{\partial \Omega^{2}}\right|_{T_{c}}\left(\Omega-\Omega_{c}\right)^{2}+O\left(\left(\Omega-\Omega_{c}\right)^{3}\right)
$$


which means $\delta=2$.

According to Eq.(3.8),

$$
\begin{aligned}
& C_{J}= \\
& -\frac{4 \pi r_{+}^{2}\left(b^{2}-a^{2} l^{2}\right)\left(-2 a l r_{+}\left(\sqrt{a^{2} l^{2} r_{+}^{2}-b^{2} r_{+}^{2}-b J l^{2}}+a l r_{+}\right)+2 b^{2} r_{+}^{2}+b J l^{2}\right)}{l\left(2 a^{3} l^{3} r_{+}^{3}+2 a^{2} l^{2} r_{+}^{2} \sqrt{a^{2} l^{2} r_{+}^{2}-b^{2} r_{+}^{2}-b J l^{2}}+b\left(J l^{2}-2 b r_{+}^{2}\right) \sqrt{a^{2} l^{2} r_{+}^{2}-b^{2} r_{+}^{2}-b J l^{2}}-2 a b^{2} l r_{+}^{3}\right)}
\end{aligned}
$$

One can set

$$
T=T_{c}(1+\epsilon), \quad r_{+}=r_{c}(1+\Delta)
$$

where $|\epsilon|,|\Delta| \ll 1$. Because the entropy $S$ and the temperature $T$ can both be expressed as $S=S\left(r_{+}, J\right), T=T\left(r_{+}, J\right)$, and

$$
C_{J}=\left.T \frac{\partial S}{\partial T}\right|_{J}=T \frac{\left.\frac{\partial S}{\partial r_{+}}\right|_{J}}{\left.\frac{\partial T}{\partial r_{+}}\right|_{J}}
$$

According to Fig.2, at the critical point $r=r_{c}$

$$
\left.\left(\frac{\partial T}{\partial r_{+}}\right)_{J}\right|_{r=r_{c}}=0
$$

Moreover one can easily verify $\left.\left(\frac{\partial^{2} T}{\partial r_{+}^{2}}\right)_{J}\right|_{r=r_{c}} \neq 0$. Therefore, in a sufficiently small neighborhood of $r_{c}$, one can expand $T$ in terms of $r_{+}$as

$$
T\left(r_{+}\right)=T\left(r_{c}\right)+\left.\frac{1}{2}\left(\frac{\partial^{2} T}{\partial r_{+}^{2}}\right)_{J}\right|_{r=r_{c}} r_{c}^{2} \Delta^{2}+O\left(\Delta^{3}\right)
$$

from which we obtain

$$
\Delta=\frac{\epsilon^{1 / 2}}{D^{1 / 2}}
$$

where

$$
D=\left.\frac{r_{c}^{2}}{2 T_{c}}\left(\frac{\partial^{2} T}{\partial r_{+}^{2}}\right)_{J}\right|_{r=r_{c}}
$$

Substitute Eq.(4.10) into Eq.(4.9), we can derive that the critical behavior of $C_{J}$ is described by

$$
C_{J} \approx \frac{A}{\epsilon^{1 / 2}}
$$

where $A$ is a function of $a, b, l, J_{c}, D$ and very complicated. Here we do not give the detailed expression. Comparing Eq.(4.16) with Eq.(4.5), one can find that $\alpha=1 / 2$.

To calculate $\beta$ we first derive the $\Omega$ as function of $r_{+}, J$.

$$
\Omega\left(r_{+}, J\right)=\frac{\sqrt{a^{2} l^{2} r_{+}^{2}-b^{2} r_{+}^{2}-b J l^{2}}+a l r_{+}}{b l r_{+}}
$$

For fixed $a, b, l$ and the critical $J_{c}$,

$$
\Omega\left(r_{+}, J\right)=\Omega\left(r_{c}, J_{c}\right)+\left.\left(\frac{\partial \Omega}{\partial r_{+}}\right)_{J}\right|_{r=r_{c}}\left(r_{+}-r_{c}\right)+\text { higher order terms }
$$


Ignoring the higher order terms, we finally obtain

$$
\Omega\left(r_{+}, J\right)-\Omega\left(r_{c}, J_{c}\right)=\frac{J_{c} l}{r_{c}^{2} \sqrt{a^{2} l^{2} r_{c}^{2}-b^{2} r_{c}^{2}-b J_{c} l^{2}} T_{c}^{1 / 2} D^{1 / 2}}\left|T-T_{c}\right|^{1 / 2}
$$

Therefore $\beta=1 / 2$.

Following the previous approach one can express the $\kappa_{T}$ as function of $r_{+}, J$. Utilizing Eq.(4.10) we can obtain

$$
\kappa_{T} \approx \frac{B}{\Delta}=\frac{B D^{1 / 2}}{\epsilon^{1 / 2}}=\frac{B D^{1 / 2 T_{c}^{1 / 2}}}{\left|T-T_{c}\right|^{1 / 2}}
$$

which means $\gamma=1 / 2$. Therefore the critical exponents $\alpha, \beta, \gamma, \delta$ have the same values as the ones obtained in the Hořava-Lifshitz black hole and the Born-Infeld black hole[41, 42]. Obviously they obey the scaling symmetry like the ordinary thermodynamic systems

$$
\begin{aligned}
& \alpha+2 \beta+\gamma=2, \quad \alpha+\beta(\delta+1)=2 \\
& \gamma(\delta+1)=(2-\alpha)(\delta-1), \quad \gamma=\beta(\delta-1)
\end{aligned}
$$

\section{DISCUSSION AND CONCLUSION}

In this paper, we adopted Ehrenfest's classification to study the phase transition of the BTZ black hole with torsion obtained in the MB topological gravitational model. Although the gravitational action contains torsion, the metric part of the BTZ black hole with torsion looks like the usual BTZ solution. Because of the existence of the Chern-Simons term and the Nieh-Yan term, the conserved charges for the BTZ black hole should be modified. Inclusion of these topological terms makes the thermodynamic properties and critical behaviors of BTZ black hole with torsion very different from the ones of the usual BTZ black hole obtained in GR.

By treating the effective cosmological constant as a thermodynamic pressure, in the extended phase space we completely followed the standard of Ehrenfest to explore the type of the phase transition of the BTZ black hole with torsion. It is shown that when $|a| l \leq|b|$ the Gibbs free energy and entropy are continuous functions of temperature, however the heat capacity $C_{P}$, the isothermal compressibility $\kappa$ and the expansion coefficient $\alpha$ are all divergent at the critical point. This means this kind of phase transition for the BTZ black hole with torsion is continuous or second order. Nevertheless, the phase transition and critical behavior are different from the ones in the van der Waals liquid/gas system. Because $a, b$ here are related to the parameters $\alpha_{3}, \alpha_{4}$ in the action of MB model. Thus whether phase transition can happen depends not only upon the black hole solutions, but also upon the gravitational actions.

Moreover we also considered the non-extended phase space. In this case, no direct thermodynamic analogy for the the isothermal compressibility exists. Thus we employed another form and named it $\kappa_{T}$. It is shown that the $\kappa_{T}$ also diverge at the same critical point. Therefore in the non-extended phase space, the phase transition is also the second order. The critical exponents for the BTZT black hole are also calculated, which are the same as the ones obtained in the Hořava-Lifshitz black hole and the Born-Infeld black hole. Is this just a coincidence, or is there some inherent reason, still need consideration further.

Although we discussed the three-dimensional topological model with torsion, the results have included the torsion-free case which corresponds to the topologically massive gravity (TMG)[43]. 
For the TMG, the field equations of which are also solved by the BTZ metric (CS-BTZ solution). The conserved charges and the entropy are modified to be [31, 44, 45]

$$
M=M_{0}-\frac{\beta}{L^{2}} J_{0}, \quad J=J_{0}-\beta M_{0}, \quad S=4 \pi\left(r_{+}-\frac{\beta}{L} r_{-}\right)
$$

Here $\beta$ is the Chern-Simons coupling constant and $L$ is the usual cosmological radius. Obviously the phase transition and the critical behaviors for the BTZ black hole in the TMG correspond to the $a=1$ case of the BTZT black hole in the MB model. Therefore when $|\beta|>L$ phase transition also exists in the CS-BTZ black hole. Similarly, in this time the mass, angular momentum and the entropy cannot all be positive.

\section{Acknowledgements}

MSM thanks H. H. Zhao and H. F. Li for useful discussion. This work is supported in part by NSFC under Grant Nos.(11247261;11175109;11075098;11205097).

[1] P. Davies, Proceedings of the Royal Society of London. A353(1977): 499-521.

[2] S. Hawking and D. N. Page, Commun. Math. Phys. 87, 577 (1983).

[3] P. Hut, Monthly Notices of the Royal Astronomical Society, 1977, 180: 379-389.

[4] L. M. Sokolowski, P. Mazur, Journal of Physics A 1980, 13(3): 1113.

[5] C. O. Lousto, Physical Review D, 1995, 51(4): 1733.

[6] C. Peca, J. P. S. Lemos, Phys. Rev. D 59, 124007 (1999).

[7] A. Chamblin, R. Emparan, C. Johnson, and R. Myers, Phys.Rev. D60 (1999) 064018, [hepth/9902170].

[8] A. Chamblin, R. Emparan, C. Johnson, and R. Myers, Phys.Rev. D60 (1999) 104026, [hepth/9904197].

[9] X. N. Wu, Physical Review D, 2000, 62(12): 124023.

[10] D. Kastor, S. Ray, J. Traschen, Class.Quant.Grav. 26(2009) 195011.

[11] B. P. Dolan, Class.Quant.Grav. 28(2011) 235017, [arXiv:1106.6260].

[12] M. Cvetic, G. Gibbons, D. Kubiznak, and C. Pope, Phys.Rev. D84 (2011) 024037, [arXiv:1012.2888].

[13] C. Niu, Y. Tian, X. N. Wu, Physical Review D, 2012, 85(2): 024017.

[14] D. Kubiznak, and R. B. Mann, JHEP 2012(2012): 1-25.

[15] S. Gunasekaran, D. Kubiznak, R. B. Mann, JHEP, 2012, (11): 1-43.

[16] M. Banados, C. Teitelboim, J. Zanelli, Physical Review Letters, 1992, 69(13): 1849;

[17] M. Banados, M Henneaux, C. Teitelboim, J. Zanelli, Physical Review D, 1993, 48(4): 1506.

[18] E. Witten, Adv. Theor. Math. Phys. 2, 505(1998); arXiv:hep-th/9803131.

[19] E. W. Mielke, P. Baekler, Physics Letters A 156(1991): 399-403.

[20] P. Baekler, E. W. Mielke, and F. W. Hehl. Il Nuovo Cimento B Series 11 107(1992): 91-110.

[21] A. A. Garcia, F. W. Hehl, C. Heinicke, A. Macias, Physical Review D 67(2003): 124016.

[22] M. Blagojević, and M. Vasilić, Phys. Rev. D 68, 104023 (2003).

[23] E.W. Mielke and A. A. R. Maggiolo, Phys. Rev. D 68, 104026 (2003). 
[24] M. Blagojević, B. Cvetković, Classical and Quantum Gravity 23(2006): 4781.

[25] M. Blagojević, B. Cvetković, Physical Review D, 2010, 81(12): 124024.

[26] M. Blagojević, B. Cvetković, O. Miskovic, et al. , JHEP, 2013, 2013(5): 1-30.

[27] Meng-Sen Ma, Ren Zhao, to be published in Phys. Rev. D.

[28] H. T. Nieh, M. L. Yan, Journal of Mathematical Physics, 1982, 23: 373; Annals of Physics, 1982, 138(2): 237-259.

[29] O. Chandia and J.Zanelli, Phys. Rev. D 55 (1997) 7580; "Torsional Topological Invariants (and their relevance for real life)", hep-th/9708138.

[30] M. Blagojević, B. Cvetković, and M. Vasilić, Phys. Rev. D88, 101501(R) (2013).

[31] S. N. Solodukhin, Physical Review D, 2006, 74(2): 024015.

[32] R. G. Cai, J. H. Cho, Physical Review D, 1999, 60(6): 067502.

[33] H. Quevedo, A. Sanchez, Physical Review D, 2009, 79(2): 024012.

[34] B. P. Dolan, Physical Review D, 2011, 84: 127503.

[35] B. P. Dolan, Class. Quant. Grav. 28(2011)125020,arXiv:1008.5023

[36] M. Cvetic, S. Noiri, S. D. Odinsov, Nucl. Phys. B628(2002) 295.

[37] T. Sarkar, G. Sengupta, B. N. Tiwari, Journal of High Energy Physics, 2006, 2006(11): 015.

[38] H. E. Stanley, "Introduction to phase transitions and critical phenomena" (Oxford University Press, New York, 1987).

[39] R. Banerjee, S. K. Modak, and S. Samanta, Phys. Rev. D84, 064024 (2011).

[40] Y. D. Tsai, X. N. Wu, and Y. Yang, Phys. Rev. D85, 044005(2012).

[41] B. R. Majhi, D. Roychowdhury, Class. Quant. Grav. 29: 245012, 2012.

[42] R. Banerjee, D. Roychowdhury, Phys. Rev. D85, 044040(2012).

[43] S. Deser, R. Jackiw, S. Templeton, Phys. Rev. Lett., 1982, 48(15): 975.

[44] M. I. Park, Physical Review D, 2008, 77(2): 026011.

[45] Paul K. Townsend, Baocheng Zhang, arXiv:1302.3874. 